\title{
FIRST COMPARISON OF REMOTE CESIUM FOUNTAINS
}

\author{
T. Parker*, P. Hetzel", S. Jefferts*, S. Weyers", L. Nelson*, A. Bauch", J. Levine* \\ * National Institute of Standards and Technology \\ 325 Broadway, Boulder, CO 80305, USA \\ \#Physikalisch-Technische Bundesanstalt, \\ POB 3345, D-38023 Braunschweig, GERMANY
}

\begin{abstract}
The frequencies of the cesium fountain primary frequency standards at the National Institute of Standards and Technology and the Physikalisch-Technische Bundesanstalt have been compared. Two-way satellite time and frequency transfer and GPS carrier-phase were the principal frequency-transfer techniques used to make the comparison. For the 15-day interval in which both fountains were in operation the frequencies were compared with an additional uncertainty due to the comparison process of only $6.2 \times 10^{-16}$. The two standards agree within their stated one-sigma uncertainties of $\sim 1.7 \times 10^{-15}$.
\end{abstract}

Key Words: atomic frequency standard, cesium fountain, comparison.

\section{Introduction}

Since August of 2000 the cesium-fountain primary frequency standards at the National Institute of Standards and Technology (NIST) and the Physikalisch-Technische Bundesanstalt (PTB) have been in service on a discontinuous basis. Significantly, during the month of August the two fountains were operated at nearly the same time. The NIST fountain, NIST-Fl, was in operation during the period MJD (Modified Julian Date) 51764 to 51794 , and the PTB fountain, CSF1, was operated over the period MJD 51764 to 51779 . This gives a 15-day overlap. There were also four additional periods of operation for the PTB standard, MJD 51799 to 51814,51824 to 51839 , 51864 to 51879 , and 52009 to 520024 . A second NIST-F1 run occurred over the interval MJD 51939 to 51969 (February 2001). Since August the NIST standard has accumulated 60 days of run time and the PTB standard 75 days. These evaluations have all been reported to the Bureau International des Poids et Mesures (BIPM), and details of the frequency standards have been presented in other papers [1], [ 2].

The nearly simultaneous operation of these two standards provided an excellent opportunity for comparison of their frequencies. The best comparison comes from the August NIST-F1 run and the first two PTB-CSF1 runs.
However, all seven runs can also be compared with somewhat increased uncertainty.

The PTB-CSF1 was operated for the first four runs at constant operational parameters, and the real measurement time comprised about $96.3 \%$ of the nominal total measurement time of 1440 hours. During the operation of NIST-F1 a range of atom densities was used, so it is not practical to shorten the comparison period to coincide exactly with that of the first interval for CSF1. Therefore, the comparison must be made by extending the PTB interval with a stable (but not necessarily accurate) frequency reference. This can be done with either EAL, which is a free atomic scale calculated by the Bureau International des Poids et Measures (BIPM), or the post processed NIST maser ensemble, ATIE. The fact that the overlap of the two fountain runs is not exact means that the stability of the frequency reference contributes to the uncertainty of the comparison. The fifth PTB-CSF1 run was made with a range of atom densities in a manner similar to that of the NIST-F1 runs.

Long-distance techniques for frequency comparison must be used since the two primary frequency standards under discussion are separated by thousands of kilometers, and this also adds an additional uncertainty to the comparison. The extrapolation and frequency-transfer uncertainties are discussed in this paper, and overall comparison uncertainties are calculated.

\section{Frequency Transfer}

Three techniques for time and frequency transfer were used for the fountain comparison in order to minimize the chance of a statistical aberration. These techniques were Two-Way Satellite Time and Frequency Transfer (TWSTFT) [3], GPS carrier-phase [4], and GPS commonview [5]. The GPS common-view comparison was made with data from the BIPM publication Circular $\mathrm{T}$, where corrections are made using precise orbits and measured ionospheric data. The TWSTFT measurements followed the standard three days per week (Monday, Wednesday and Friday) BIPM schedule and were made at $\mathrm{Ku}$-band using a commercial communications satellite. The two-way data used for the fountain comparison were the same as that reported to the BIPM, except that data comparing 
UTC(NIST) to the maser $\mathrm{H} 2$ at PTB was extracted. The GPS carrier-phase data comes from two dual-frequency, geodetic-quality receivers located at NIST and PTB [6]. The TWSTFT and carrier-phase data both give the time difference between UTC(NIST) (which is derived from a maser ensemble) and the maser $\mathrm{H} 2$. The fountain frequencies can be related to these two standards via internal measurements. At PTB the fountain directly measures the frequency of $\mathrm{H} 2$. At NIST an internal measurement system is used to relate the frequency of the specific maser used as the fountain reference to UTC(NIST). The uncertainty of the NIST internal measurement is well under $1 \times 10^{-16}$ at 15 days. The common-view GPS data relate the fountain frequencies to International Atomic Time (TAI) via the reference clocks for each standard.

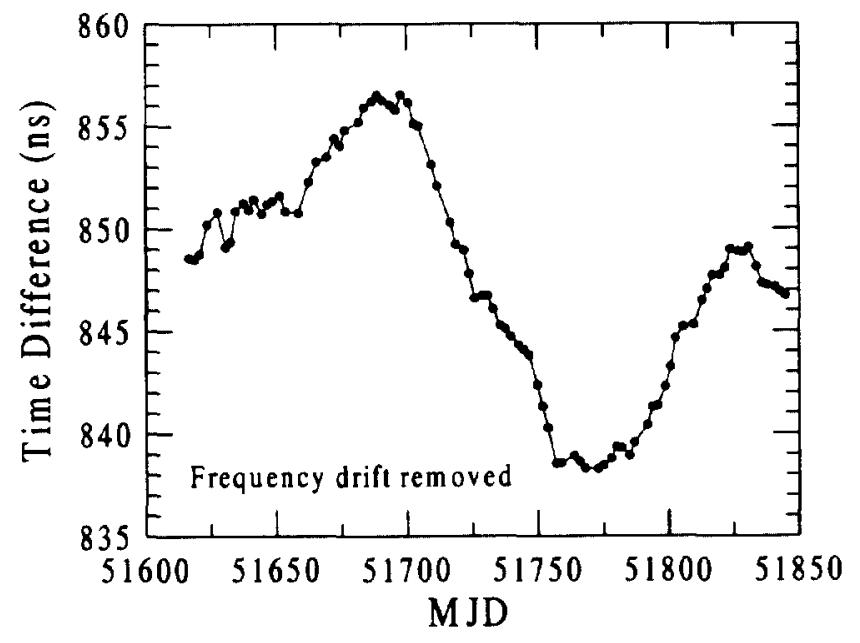

Figure 1. Time difference between UTC(NIST) and $\mathrm{H} 2$ via the two-way method.

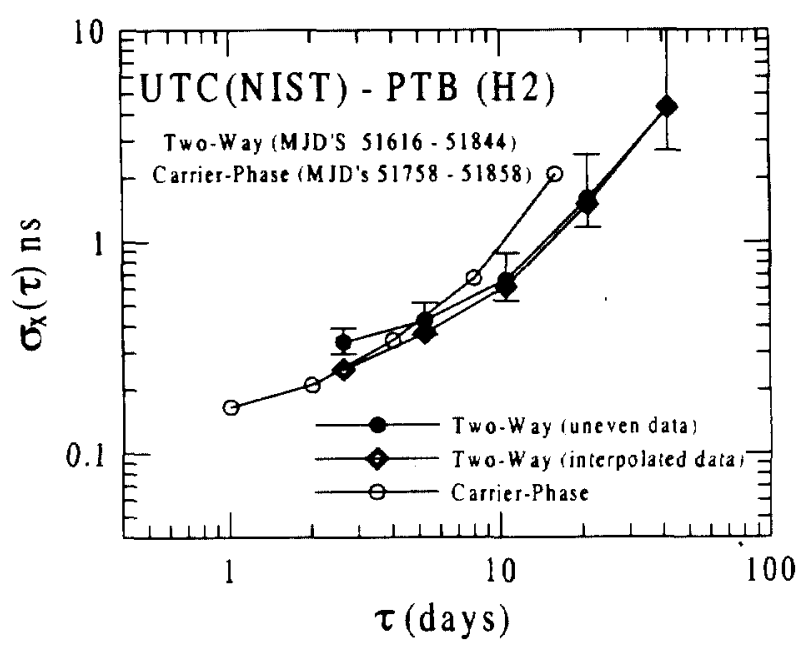

Figure 2. Time deviation of UTC(NIST) minus H2.
Figure 1 shows time-difference data for UTC(NIST) H2 via the TWSTFT link for a 200 day period that includes most of the fountain runs. Frequency offset and drift have been removed. It is evident in Fig. 1 that the day-to-day variations are typically less than $1 \mathrm{~ns}$. The larger long-term instabilities are due to clock noise. Figure 2 shows that the time deviation, $\sigma_{x}(\tau)$, for the two-way data is about $300 \mathrm{ps}$ at a few days. It is very likely that even at a few days the time deviations are also influenced to some extent by clock noise. $\sigma_{x}(\tau)$ was calculated for both the unevenly spaced two-way data (solid circles) and for data interpolated to an even spacing (diamonds). If the time difference data had been taken with an even spacing $\sigma_{x}(\tau)$ would fall between the two curves. Figure 2 also shows time deviations for the carrier-phase comparison. At a few days the carrier-phase stability appears to be slightly better than that of two-way. The higher noise level for the carrier-phase data at $\tau=15$ days is most likely just a consequence of the shorter analysis interval.

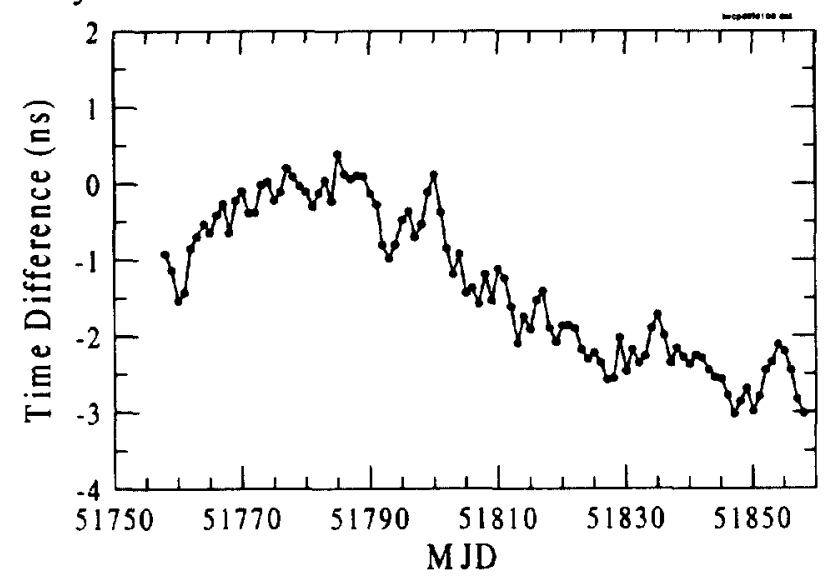

Figure 3. TWSTFT minus GPS carrier-phase for UTC(NIST) - H2

By differencing the data from both transfer techniques the clock noise can be removed, giving a clearer picture of the stability of the frequency-transfer processes, particularly in the long term. Figure 3 shows the time difference between the TWSTFT and carrier-phase data for the UTC(NIST) - H2 link over a 100-day period that includes the August NIST-F1 run and the first three PTB-CSF1 runs. The data interval is one day and the two-way data have been interpolated to match this interval. As expected, the long-term stability of this data is better than that in Fig. 1, but there is still some drift in the time difference. It is not clear whether this drift comes from two-way, carrier-phase, or both, but in any case, it would constitute a real systematic error (rate offset) on the order of $5 \times 10^{-16}$ in the frequency measurement if attributed to either one of the methods. It is important to note that the final carrier-phase solution is a combination of 3.5-day analysis periods with half-day overlaps. Therefore the solution is sensitive to the overlapping offsets of the consecutive data series as well as to corrections for jumps and gaps in the data. This could be 
a possible cause of the drift in the time difference. Another possibility is seasonal temperature fluctuations affecting either system.

Figure 4 shows the time deviation for this data and Fig. 5 shows the Allan deviation. The $\sigma_{x}(\tau)$ and Allan deviation values at one day are both biased low because of the interpolation of the two-way data, but in general the time deviation is flicker-phase in nature at a level of about 200 ps. The Allan deviation plot indicates that the combined frequency uncertainty of TWSTFT and carrier phase is about $5 \times 10^{-16}$ at 15 days. However, this may be optimistic because both the Allan deviation and time deviation statistics are based on the second difference of a time series, which is insensitive to a rate (or frequency) offset.

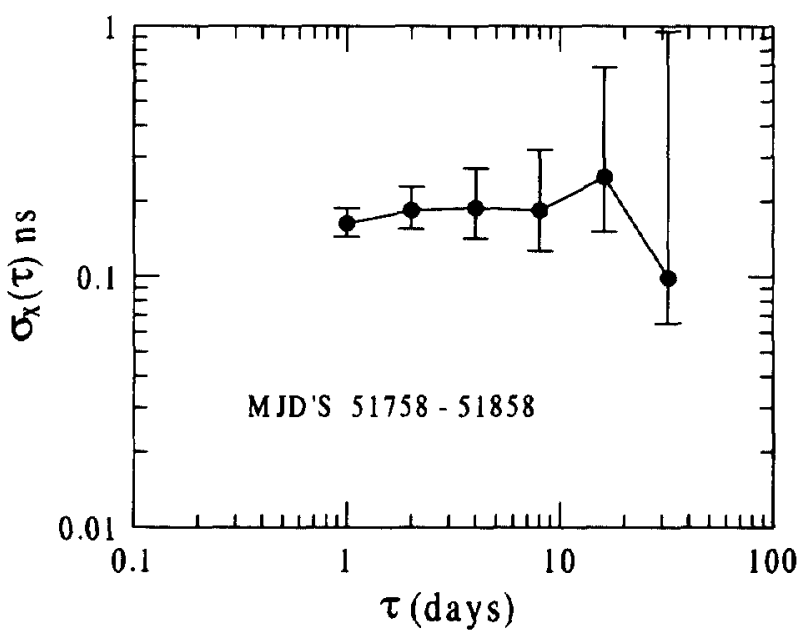

Figure 4. Time deviation of TWSTFT minus carrier-phase for UTC(NIST) - H2

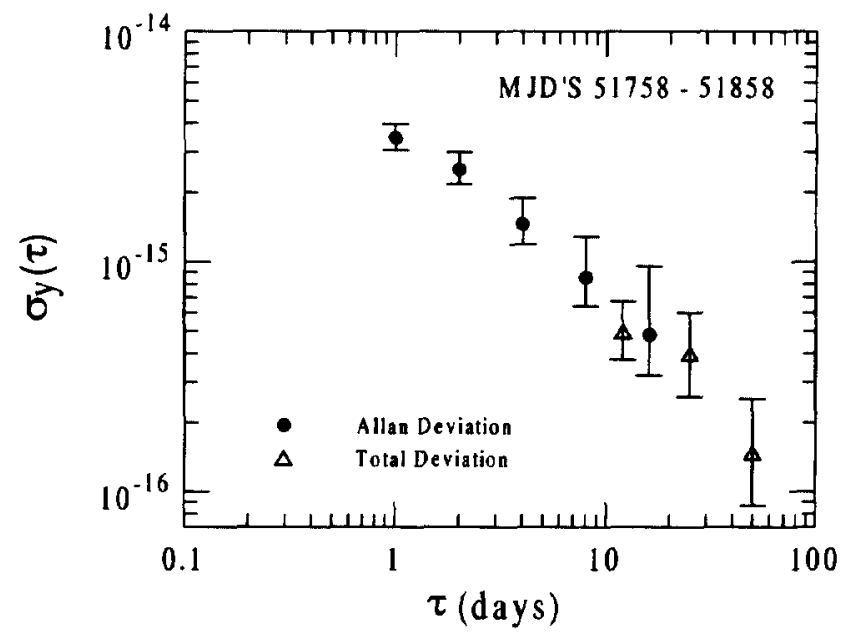

Figure 5. Allan deviation of TWSTFT minus carrier-phase for UTC(NIST)-H2
A slightly larger uncertainty of $6 \times 10^{-16}$ at 15 days is obtained using a first-difference statistic that is the RMS fractional frequency of the time-series data in Fig. 3 [7]. This is more consistent with the observed slope in the data. For the purposes of this comparison we will assume that the instabilities of TWSTFT and carrier phase are independent and that they contribute equally to the combined instability of $6 \times 10^{-16}$. This gives a frequency-transfer uncertainty of $4.2 \times 10^{-16}$ at 15 days for each of the two techniques individually.

For the uncertainty in the GPS common-view data we use the BIPM estimate of $2 \times 10^{-15}$ at 15 days, as stated in Circular T.

\section{Extrapolation}

A stable frequency reference must be used as a transfer standard since none of the fountain evaluations overlap perfectly. To estimate the uncertainty of comparisons with dead time we have used the method of Douglas and Boulanger [8]. The comparisons were made with both ATIE [9], [10], a post-processed scale based on a maser ensemble at NIST, and EAL, the free atomic scale calculated at the BIPM. The stability characteristics of these scales are shown in Table 1 for $\tau$ in units of 1 day.

Table 1. Frequency Stability Characteristics of AT $1 E$ and EAL

\begin{tabular}{|c|c|c|c|}
\hline Scale & White FM & Flicker FM & RW FM \\
\hline AT1E & $4 \times 10^{-16}\left(\tau^{-1 / 2}\right)$ & $4 \times 10^{-16}$ & $1.3 \times 10^{-16}\left(\tau^{1 / 2}\right)$ \\
\hline EAI & $60 \times 10^{-16}\left(\tau^{-12}\right)$ & $6 \times 10^{-16}$ & $1.6 \times 10^{-16}\left(\tau^{1 / 2}\right)$ \\
\hline
\end{tabular}

The white FM and flicker FM noise characteristics of AT1E were estimated from internal measurements made at NIST, and the random-walk FM noise level was estimated from measurements against EAL and CS2 (a thermal-beam primary frequency standard at PTB). See [10] for a more detailed discussion. The stability characteristics of EAL are those published by the BIPM in Circular T. The main advantage of AT1E is its much lower white FM noise. This is expected since EAL is affected by noise from GPS common-view.

\section{Comparison Results}

The best candidates for comparison are the August NIST-F1 run and the first three PTB-CSF1 runs because they have the closest overlap. The results are summarized in Tables 2 a-d. The stated uncertainties for the August NIST-F1 run are $u b=1.5 \times 10^{-15}$ and $u a=0.8 \times 10^{-15}$, where $\mathrm{ub}$ is the systematic uncertainty and ua is the statistical uncertainty. The combined uncertainty for this NIST-F1 run is $1.7 \times 10^{-15}$. (For the second NIST-F1 evaluation the combined uncertainty is $1.8 \times 10^{-15}$.) Since the uncertainties 
for the first three PTB-CSF1 runs were not all the same they are listed individually in the tables. All uncertainties that are statistical in nature will be identified as "ua". The frequency uncertainty due to the time-transfer process is identified as ua(TT) and ranges from $0.42 \times 10^{-15}$ for TWSTFT and carrier-phase to $2.0 \times 10^{-15}$ for common-view GPS. The uncertainties due to dead time are identified as ua(dead) and range from $0.46 \times 10^{-15}$ to $2.9 \times 10^{-15}$. The total uncertainty due to the comparison process is ua(comp.), which is obtained from ua(TT) and ua(dead) combined in quadrature. Finally, the uncertainty of the remote standard, $\mathrm{u}$ (remote), is calculated from the quadrature combination of ua(standard), ub(standard), and ua(comp.). In this discussion PTB-CSFI will be treated as the remote standard as seen from NIST, although the roles could be reversed.

Table 2. Comparison of NIST-F1 with PTB-CSF1 MJD 51764 - 51839

Table 2a Referenced to AT1E via two-way (units of $10^{-15}$ )

$\mathrm{ua}(\mathrm{TT})=0.42$

\begin{tabular}{|c|c|c|c|c|c|c|c|c|c|}
\hline $\begin{array}{c}\text { PTB } \\
\text { Runs }\end{array}$ & $y$ (F1-CSF1) & $\begin{array}{c}\text { ub } \\
\text { CSF1 }\end{array}$ & $\begin{array}{c}\text { ua } \\
\text { CSF1 }\end{array}$ & $\begin{array}{c}\text { CSF1 } \\
\text { CSF }\end{array}$ & $\begin{array}{c}\text { ua } \\
\text { (dead) }\end{array}$ & $\begin{array}{c}\text { ua } \\
\text { (comp.) }\end{array}$ & $\begin{array}{c}\text { u } \\
\text { (remote) }\end{array}$ & $\begin{array}{c}\text { (F1-CSF1) } \\
\text { (w.avg.) }\end{array}$ & $\begin{array}{c}\text { uw } \\
\text { (remote) }\end{array}$ \\
\hline First & -0.36 & 1.5 & 1.0 & 1.8 & 0.46 & 0.62 & 1.9 & -0.36 & 1.9 (1 run) \\
\hline Second & 4.23 & 1.4 & 1.0 & 1.7 & 1.2 & 1.3 & 2.1 & 1.23 & $1.8(2$ runs) \\
\hline Third & 2.13 & 1.4 & 1.0 & 1.7 & 1.7 & 1.8 & 2.5 & $1.39(?)$ & $1.7(3$ runs) \\
\hline
\end{tabular}

Table $2 b$ Referenced to ATIE via GPS carrier phase (units of $10^{-15}$ )

$\mathrm{ua}(\mathrm{TT})=0.42$

\begin{tabular}{|c|c|c|c|c|c|c|c|c|c|}
\hline $\begin{array}{c}\text { PTB } \\
\text { Runs }\end{array}$ & $y(F 1-C S F 1)$ & $\begin{array}{c}u b \\
\text { CSF1 }\end{array}$ & $\begin{array}{c}\text { ua } \\
\text { CSF1 }\end{array}$ & $\begin{array}{c}\text { u } \\
\text { CSF1 }\end{array}$ & $\begin{array}{c}\text { ua } \\
\text { (dead) }\end{array}$ & $\begin{array}{c}\text { ua } \\
\text { (comp.) }\end{array}$ & $\begin{array}{c}\text { u } \\
\text { (remote) }\end{array}$ & $\begin{array}{c}y \text { (F1-CSF1) } \\
\text { (w. avg.) }\end{array}$ & $\begin{array}{c}\text { uw } \\
\text { (remote) }\end{array}$ \\
\hline First & 0.03 & 1.5 & 1.0 & 1.8 & 0.46 & 0.62 & 1.9 & 0.03 & $1.9(1$ run) \\
\hline Second & 2.97 & 1.4 & 1.0 & 1.7 & 1.2 & 1.3 & 2.1 & 1.05 & $1.8(2$ runs) \\
\hline Third & 2.15 & 1.4 & 1.0 & 1.7 & 1.7 & 1.8 & 2.5 & $1.25(?)$ & $1.7(3$ runs) \\
\hline
\end{tabular}

Table 2c Referenced to AT 1E via common-view GPS from Circular T (units of $10^{-15}$ ) $u a(T T)=2.0$

\begin{tabular}{|c|c|c|c|c|c|c|c|c|c|}
\hline $\begin{array}{c}\text { PTB } \\
\text { Runs }\end{array}$ & $\mathrm{y}(\mathrm{F} 1-\mathrm{CSF} 1)$ & $\begin{array}{c}\mathrm{ub} \\
\mathrm{CSF}\end{array}$ & $\begin{array}{c}\mathrm{ua} \\
\mathrm{CSF} 1\end{array}$ & $\begin{array}{c}\mathrm{u} \\
\mathrm{CSF} 1\end{array}$ & $\begin{array}{c}\mathrm{ua} \\
\text { (dead) }\end{array}$ & $\begin{array}{c}\mathrm{ua} \\
\text { (comp.) }\end{array}$ & $\begin{array}{c}\mathrm{u} \\
\text { (remote) }\end{array}$ & $\begin{array}{c}\mathrm{y} \text { (F1-CSF1) } \\
\text { (w. avg.) }\end{array}$ & $\begin{array}{c}\text { uw } \\
\text { (remote) }\end{array}$ \\
\hline First & -1.63 & 1.5 & 1.0 & 1.8 & 0.46 & 2.0 & 2.7 & -1.63 & 2.7 (1 run) \\
\hline Second & 5.20 & 1.4 & 1.0 & 1.7 & 1.2 & 2.3 & 2.9 & 1.42 & 2.2 (2 runs) \\
\hline Third & 2.20 & 1.4 & 1.0 & 1.7 & 1.7 & 2.6 & 3.1 & $1.63(?)$ & 2.0 (3 runs) \\
\hline
\end{tabular}

Table 2d Referenced to EAL via common-view GPS from Circular T (units of $10^{-15}$ ) ua(TT) $=2.0$

\begin{tabular}{|c|c|c|c|c|c|c|c|c|c|}
\hline $\begin{array}{c}\text { PTB } \\
\text { Runs }\end{array}$ & $\mathrm{y}(\mathrm{F} 1-\mathrm{CSF} 1)$ & $\begin{array}{c}\mathrm{ub} \\
\mathrm{CSF1}\end{array}$ & $\begin{array}{c}\mathrm{ua} \\
\mathrm{CSF} 1\end{array}$ & $\begin{array}{c}\mathrm{u} \\
\mathrm{CSF} 1\end{array}$ & $\begin{array}{c}\mathrm{ua} \\
\text { (dead) }\end{array}$ & $\begin{array}{c}\text { ua } \\
\text { (comp.) }\end{array}$ & $\begin{array}{c}\mathrm{u} \\
\text { (remote) }\end{array}$ & $\begin{array}{c}\mathrm{y}(\mathrm{F} 1-\mathrm{CSF} \text { () } \\
\text { (w. avg.) }\end{array}$ & $\begin{array}{c}\text { uw } \\
\text { (remote) }\end{array}$ \\
\hline First & 2.5 & 1.5 & 1.0 & 1.8 & 1.2 & 2.3 & 2.9 & 2.5 & $2.9(1 \mathrm{run})$ \\
\hline Second & 4.3 & 1.4 & 1.0 & 1.7 & 2.5 & 3.2 & 3.6 & 3.16 & $2.5(2$ runs) \\
\hline Third & 0.2 & 1.4 & 1.0 & 1.7 & 2.9 & 3.5 & 3.9 & $2.46(?)$ & $2.3(3$ runs) \\
\hline
\end{tabular}

The four tables compare data for: (a) TWSTFT with ATIE used as a transfer standard, (b) GPS carrier-phase with ATIE used as a transfer standard, (c) GPS commonview with ATIE used as a transfer standard, and (d) GPS common-view with EAL used as a transfer standard. All uncertainties are one sigma. The first row of data in each table is for the first PTB run, which overlapped the NIST run by 15 days. In Tables 2 a-c, the uncertainty ua(dead) in 
this row is small (but not zero) because the two runs overlapped, but were not the same length. Note that ua(dead) is considerably larger with EAL used as the frequency reference (Table 2d) since EAL has a much larger white FM noise level. The observed values for the fractional frequency difference of the two standards, $y(F 1-C S F 1)$, are $-0.36 \times 10^{-15}$ for comparison by two-way, and $0.03 \times 10^{-15}$ for comparison by carrier-phase. ua(comp.) is $0.62 \times 10^{-15}$ for both methods and u(remote), the uncertainty of PTB-CSF1 as seen from NIST, is $1.9 \times 10^{-15}$. This is only slightly larger than the stated combined uncertainty of CSF1, which is $1.8 \times 10^{-15}$ for that run. The two standards are in excellent agreement in the first run. The comparison results using common-view GPS with ATIE and EAL are given in the first data rows of Tables $2 \mathrm{c}$ and $2 \mathrm{~d}$. Note that ua(comp.) is much larger. The agreement between the two standards is not as good as with two-way and carrier phase, but is still within $u$ (remote) for these comparisons.

The second and third runs of PTB-CSF1 can also be compared to the August NIST-F1 run, as shown in the second and third rows of the tables. However, ua(dead) gets larger because the runs don't overlap at all. In general, the agreement in the second run is not as good as in the first. In the second run the frequency differences for the comparisons using AT1E with two-way and AT1E with common-view are large enough that the error bars of the two standards don't overlap. For ATIE with carrier-phase and EAL with common-view they do overlap. The agreement in the third run is much better, even though $\mathrm{u}$ (remote) is getting rather large, particularly when EAL and common-view are used.

A weighted average of the various runs can also be calculated. The last two columns in rows 2 and 3 show the weighted averages for $y(F 1-C S F 1)$ and the corresponding uncertainties, uw(remote), for the first two runs, and all three runs, respectively. (Results for the first run are repeated for clarity in the first row of these columns even though there is no averaging.) The weighting was based on the combined statistical uncertainties for each case. It is questionable how meaningful the weighted average is for all three runs because it is very likely that the errors due to the dead time are correlated between the second and third runs; thus the question marks in the tables. However, the weighted average of the first two runs is meaningful, and it shows that the fountains agree within $\sim 1.2 \times 10^{-15}$ for either the TWSTFT or carrier-phase comparisons. Note that uw(remote) for the weighted average of the first and second runs using these two comparison techniques, $1.8 \times 10^{-15}$, is very close to the level of the stated uncertainty of the PTBCSF1.

In principle, one could average the TWSTFT and carrier-phase comparisons together to further reduce ua(TT) by another factor of $1 / \sqrt{ } 2$. However, this would have only a small impact, and may not be justified until the assumption of independence between TWSTFT and carrier-phase can be verified.

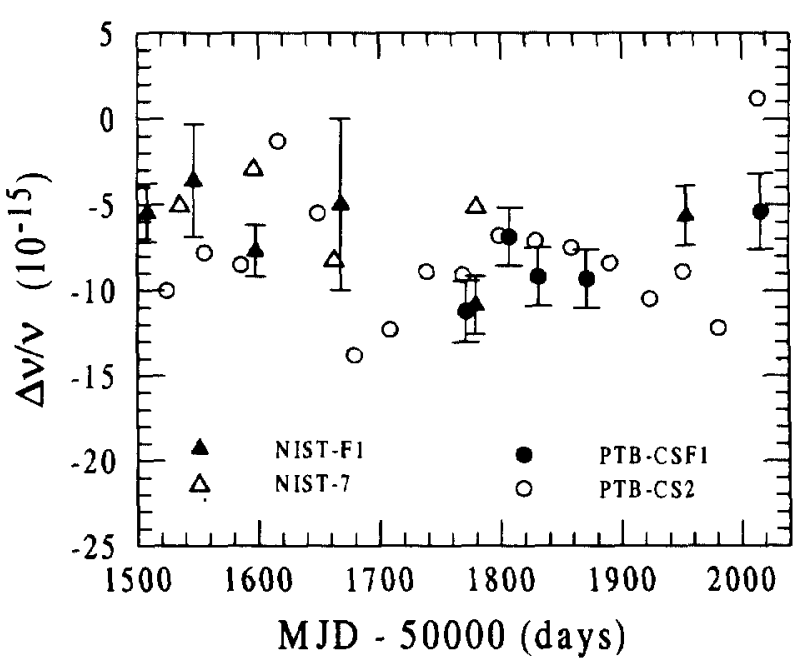

Figure 6. Fractional frequency deviation of ATIE versus four primary frequency standards.

Figure 6 shows the fractional frequency difference of AT 1E (with an arbitrary frequency offset removed) relative to four of the best primary frequency standards over a 500day period. The standards include the two fountains discussed in this paper and two thermal-beam standards, NIST-7 and PTB-CS2. The figure contains all available fountain evaluations (formal and informal) for this period and provides a qualitative view of the relative frequencies of the four primary frequency standards. The combined uncertainty for PTB-CS2 is typically $12 \times 10^{-15}$, and for NIST-7 ranges from 5 to $10 \times 10^{-15}$. Individual uncertainties are shown for the fountains. The overall agreement between the four standards is quite good.

Though the scatter among the fountain data is large enough that a few of the error bars don't overlap, it is impossible to tell whether the fluctuations are from the standards or from ATIE. However, the scatter is statistically consistent at the one-sigma level with the uncertainties of the standards and the noise levels of ATIE [10]. From the data in Fig. 6 the average frequency difference for the two NIST-F1 evaluations since August 2000 (MJD 51764) and the five PTB-CSF1 runs is $y(F 1-C S F 1)=-0.2 \times 10^{-15}$. The uncertainty for this comparison is difficult to calculate rigorously but $\mathrm{u}$ (remote) is estimated to be in the range of $2.0 \times 10^{-15}$ to $2.6 \times 10^{-15}$. The slight downward frequency drift in Fig. 6 is a characteristic of AT1E.

Figure 7 is a similar plot that shows the fractional deviation $d$ (in seconds) of the scale interval of TAI (as obtained from Circular $T$ ) relative to the same four primary frequency standards. Note that $d=-\Delta v / v$. The details are somewhat different, but the general relationship of the four standards is about the same as that in Fig. 6. The average frequency difference for the two NIST-Fl evaluations and the five PTB-CSFI runs using TAI as the reference is 
$y(F 1-C S F 1)=0.3 \times 10^{-15}$, with an estimated range for $u\left(\right.$ remote) of $2.6 \times 10^{-15}$ to $3.3 \times 10^{-15}$.

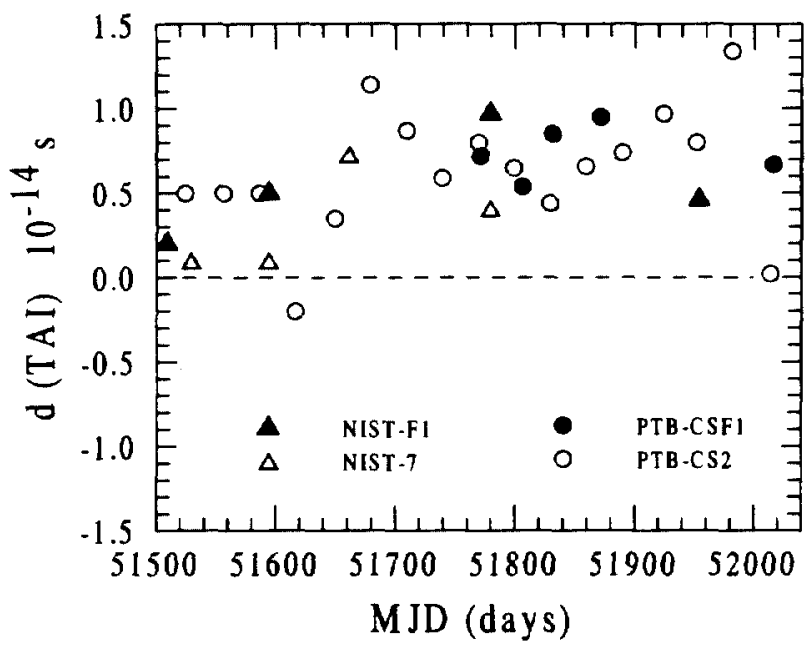

Figure 7. Fractional deviation of the duration of TAI relative to four primary frequency standards.

\section{Conclusions}

The cesium fountain primary frequency standards at PTB and NIST have been compared by three different frequency transfer techniques and with two different stable frequency references. This variety of comparison techniques was used in order to minimize the chance that a statistical fluctuation in one technique might give an unusually good or bad result. It has been demonstrated that the uncertainties of the comparison process can be reduced to a nearly negligible level with the use of TWSTFT or GPS carrier-phase if the duration of the comparison is 15 days or longer. For the data in Table 2, only two cases out of the twelve individual comparisons and the four weighted averages exhibit a frequency difference large enough that the uncertainty limits of the two standards don't overlap. This is entirely consistent with one-sigma uncertainties. In most cases the observed frequency differences are well within the uncertainties of a single standard.

The best estimate of the frequency difference of the two standards is given by the weighted average of the first two PTB runs relative to the August NIST-F1 run using either two-way or GPS carrier-phase. The frequency difference is $\sim 1.2 \times 10^{-15}$, which is well within the stated combined uncertainties of the two standards, which are $1.7 \times 10^{-15}$. A somewhat less rigorous comparison using the two most recent NIST-Fl evaluations and all five PTBCSFl runs also shows agreement well within the estimated uncertainties.

\section{References}

1 S. Weyers, R. Schröder, and A. Bauch, "Recent Results from PTB's Caesium Fountain CSF1," in Proc. $15^{\text {th }}$ European Freq. And Time Forum, to be published.

2 S. Jefferts, D. M. Meekhof, T. P. Heavner, T. E. Parker, "Latest Results from NIST-F1, a LaserCooled Cesium Fountain Primary Frequency Standard," in Proc. $15^{\text {th }}$ European Freq. And Time Forum, to be published.

3 D. Kirchner, "Two-Way Time Transfer Via Communication Satellites," in Proc. of the IEEE, vol. 79, pp. 983-990, 1991.

4 K. M. Larson and J. Levine, "Carrier-Phase Time Transfer," in IEEE Trans. on Ultrason., Ferroelect., and Freq. Contr., vol. 46, no. 4, pp. 1001-1012, 1999.

5 W. Lewandowski and C. Thomas, "GPS Time Transfer," in Proc. of the IEEE, vol. 79, pp. 991-1000, 1991.

6 L. Nelson, J. Levine and P Hetzel, "Comparing Primary Frequency Standards at NIST and PTB," in Proc. 2000 IEEE International Freq. Control Symp., pp. 622-628, 2000.

7 T. E. Parker, D. A. Howe and M. Weiss, "Accurate Frequency Comparisons at the $1 \times 10^{-15}$ Level," in Proc. 1998 IEEE International Freq. Control Symp., pp. 265-272, 1998.

8 R. J. Douglas and J. S. Boulanger, "Standard Uncertainty for Average Frequency Traceability," in Proc. $11^{\text {th }}$ European Freq. and Time Forum, pp. 345349, 1997.

9 T. E. Parker, "Hydrogen Maser Ensemble Performance and Characterization of Frequency Standards," in Proc. 1999 Joint Meeting of the European Freq. and Time Forum and the IEEE International Freq. Control Symp., pp. 173-176, 1999.

10 T. E. Parker, "Comparing and Evaluating the Performance of Primary Frequency Standards: Impact of Dead Time," in this Proceedings. 